\title{
SOME THEOREMS ON BOUNDED HOLOMORPHIC FUNCTIONS
}

\author{
BY E. L. STOUT ${ }^{1}$ \\ Communicated by W. Rudin, January 20, 1964
}

The purpose of this note is the announcement of some results in the theory of bounded holomorphic functions on finite open Riemann surfaces. The proofs are too long to be included; they will be published elsewhere. Special cases of some of the results of this note are to be found in [4].

We consider a fixed finite open Riemann surface $R$. We thus assume that $R$ is contained as an open set in some compact Riemann surface $R_{0}$ and that $R_{0} \backslash R$ consists of finitely many topological bordered surfaces. We assume further that $\partial R$, the boundary of $R$ with respect to $R_{0}$, consists of the $m$ analytic simple closed curves $\Gamma_{1}, \cdots, \Gamma_{m}$.

Denote by $H_{\infty}[R]$ the algebra of all functions holomorphic and bounded on $R$. Given the norm $\|\cdot\|_{R}$ defined by

$$
\|f\|_{R}=\sup \{|f(\zeta)|: \zeta \in R\},
$$

$H_{\infty}[R]$ is a Banach algebra. We shall denote by $\mathfrak{M}$ the maximal ideal space of $H_{\infty}[R]$; $\mathfrak{M}$ will be regarded as the space of all nonzero complex homomorphisms together with the weak* topology. There is a natural embedding of $R$ in $\mathfrak{M}$ given by $\zeta \rightarrow \phi_{\zeta}$ where $\phi_{\zeta}(f)=f(\zeta)$ for all $f \in H_{\infty}[R]$ and all $\zeta \in R$. The assumptions on the structure of $R$ guarantee that this correspondence is a homeomorphism.

Our first result is

THEOREM 1. Let $f_{1}, \cdots, f_{n}$ be elements of $H_{\infty}[R]$ which satisfy $\left|f_{1}(\zeta)\right|+\cdots+\left|f_{n}(\zeta)\right| \geqq \delta$ for some $\delta>0$. Then there exist $g_{1}, \cdots, g_{n}$ $\in H_{\infty}[R]$ such that $f_{1}(\zeta) g_{1}(\zeta)+\cdots+f_{n}(\zeta) g_{n}(\zeta)=1$ for all $\zeta \in R$.

As is known [3, p. 163], this theorem is equivalent to the assertion that $R$ is dense in $\mathfrak{M}$ in the sense that the set of all homomorphisms of form $\phi_{5}$ is dense in $\mathfrak{M}$. It is this latter fact that our proof establishes. We first obtain the result for the case that $R$ is an annulus. The proof then proceeds along the general lines of the proof of Theorem $B$ of [4]. A different proof of Theorem 1 has been given in [1].

Making use of Theorem 1 in the form of the density of $R$ in $\mathfrak{M}$ and of certain of the constructions in the proof, we are able to obtain some

1 The research for this note was supported by National Science Foundation Grant GP 2235. 
results on interpolation in $R$. The question considered is the following: Suppose given a set $S$ of points in $R$. Under what conditions is it the case that for all bounded complex valued functions $\alpha$ on $S$ there is $f \in H_{\infty}[R]$ such that for all $\zeta \in S, f(\zeta)=\alpha(\zeta)$ ? If $S$ has this property, it is termed an interpolation set for $R$. Plainly, interpolation sets are countable.

There are several characterizations of interpolating sets. The first may be formulated in the following way. Let $\Gamma_{j}^{\prime}$ be a simple closed curve in $R$ which, together with $\Gamma_{j}$, bounds an annulus $A_{j}$ in $R$. We write $S=S_{1} \cup \ldots \cup S_{m}$ where we assume that for each $j, S_{j} \subset A_{j}$. We can then prove

THEOREM 2. The set $S$ is an interpolating set for $R$ if and only if, for each $j$, the set $S_{j}$ is one for $A_{j}$.

The proof of this theorem is in two steps. We first establish the result for the case of an annulus. Using the annular case together with the density of $R$ in $\mathfrak{M}$ and a theorem of Hoffman [3, p. 205], we are able to extend the result to the case of the general open, finite Riemann surface.

An alternative characterization of interpolation sets in $R$ is contained in

THEOREM 3. The set $\left\{\zeta_{n}\right\}$ is an interpolation set for $R$ if and only if there is a $\delta>0$ such that for all $n$ we have

$$
\delta \leqq \sup \left\{\left|f\left(\zeta_{n}\right)\right|: f \in H_{\infty}[R],\|f\|_{R} \leqq 1, f\left(\zeta_{k}\right)=0 \text { for } k \neq n\right\} .
$$

This theorem is a generalization to $R$ of the result obtained by L. Carleson [2, p. 203] in the case that $R$ is the unit disc.

If $\zeta_{0} \in R$, denote by $g\left(\cdot, \zeta_{0}\right)$ the Green's function of $R$ with singularity at $\zeta_{0}$. Theorem 2 can be used to prove

THEOREM 4. The set $S$ is an interpolation set for $R$ if and only if there exists an $M$ such that

$$
\sum_{\zeta \in S_{i} \zeta \neq \xi_{0}} g\left(\zeta, \zeta_{0}\right) \leqq M
$$

holds for all $\zeta_{0} \in S$.

For the planar case, this result is to be found in [2].

Two further theorems which are closely related to Theorems 2 and 3 are as follows. Suppose that $U$ is the open unit disc. The uniformization theorem enables us to realize $U$ as the universal covering surface of $R$ by means of an analytic map $\Phi: U \rightarrow R$. Let $G$ be the corresponding group of covering transformations. Thus $G$ is a group of con- 
formal maps of $U$ onto itself. The problem we consider is that o relating interpolation sets in $R$ to interpolation sets in $U$ by means o the map $\Phi$. We have

Theorem 5. Let $S$ be an interpolation set in $R$, and let $\Phi^{-1}[S$ $=\{z: z \in U, \Phi(z) \in S\}$. Then $\Phi^{-1}[S]$ is an interpolation set for $U$.

A converse of Theorem 5 is also valid.

THEOREM 6. Suppose that $S$ is an interpolation set for $U$ which is invariant under the group $G$ in the sense that $g[S]=S$ for all $g \in G$. Then the set $\Phi[S]$ is an interpolation set for $R$.

\section{REFERENCES}

1. N. Alling, A proof of the corona conjecture for finite open Riemann surfaces, Bull. Amer. Math. Soc. 70 (1964), 110-112.

2. L. Carleson, Research on interpolation problems, Tech. Rep., Department of Mathematics, Uppsala University, Uppsala, 1962.

3. K. Hoffman, Banach spaces of analytic functions, Prentice-Hall, Englewood Cliffs, N. J., 1962.

4. E. L. Stout, Two theorems concerning functions holomorphic on multiply connected domains, Bull. Amer. Math. Soc. 69 (1963), 527-530.

UNIVERSITY OF WISCONSIN 